Journal of Computer Science 6 (6): 634-640, 2010

ISSN 1549-3636

(C) 2010 Science Publications

\title{
DWT to Classify Automatically the Placental Tissues Development: Neural Network Approach
}

\author{
${ }^{1}$ Mohammad Ayache, ${ }^{2}$ Mohamad Khalil and ${ }^{3}$ Francois Tranquart \\ ${ }^{1}$ Department of Biomedical, Faculty of Engineering, Islamic University of Lebanon, \\ BP 30014, Highway Khalde, Lebanon \\ ${ }^{2}$ Faculty of Engineering, Lebanese University, Beirut, Lebanon \\ ${ }^{3}$ Faculty of Medicine, INSERM U930, University of Tours, France
}

\begin{abstract}
Problem statement: This study proposed an approach for classification of placental tissues development using ultrasound images. Approach: This approach was based to the selection of tissues, feature extraction by discrete wavelet transform and classification by neural network and especially the Multi Layer Perceptron (MLP). Results: The proposed approach was tested for ultrasound placental images; resulting in 95\% success rate. Conclusion/Recommendations: The method showed a good recognition for placental tissues and will be useful for detection of the placental anomalies those concerning the premature birth and the intrauterine growth retardation.
\end{abstract}

Key words: Placenta, wavelet transform, neural network, MLP

\section{INTRODUCTION}

An ultrasound diagnostic system has become an important and popular diagnostic tool since it has a wide range of applications. Specifically, due to its noninvasive and non-destructive nature, the ultrasound diagnostic system has been extensively used in the medical profession. Modern high-performance ultrasound diagnostic systems and techniques are commonly used to produce two or three-dimensional images of internal features of an object (e.g., human organs). The ultrasound diagnostic system generally uses a probe containing a wide bandwidth transducer to transmit and receive ultrasound signals. The ultrasound diagnostic system forms images of human internal tissues by electrically exciting an acoustic transducer element or an array of acoustic transducer elements to generate ultrasound signals that travel into the body. The ultrasound signals produce ultrasound echo signals since they are reflected from body tissues, which appear as discontinuities to the propagating ultrasound signals. Various ultrasound echo signals return to the transducer element and are converted into electrical signals, which are amplified and processed to produce ultrasound data for an ultrasound image of the tissues.

Ultrasonography is generally considered a safe imaging modality. However slight detrimental effects have been occasionally observed. Diagnostic ultrasound studies of the fetus are generally considered to be safe during pregnancy. However, it is difficult to differentiate between normal and abnormal tissues on the basis of ultrasound images from the placenta.

The placenta is a temporary internal organ joining the mother and fetus. The placenta transfers oxygen and nutrients from the mother to the fetus and permits the release of carbon dioxide and waste products from the fetus. The goal of our work is to study the transfer function of placental tissues during grades development using ultrasound images. Grannum and Hobbins (1982) proposed a classification of the placental maturation by ultrasound techniques. According to a visual approach, he classified the placenta into four different grades.

The aim of the present study is to propose a classification approach based on image processing techniques, in order to identify automatically different grades of the placenta. In the literature, there are different methods that can be used to extract the images in order to make the diagnosis. Wavelet transforms (Mallat, 1989) Fourier transform (Feleppa et al., 1996), discrete cosine transform (Kurnaz et al., 2001; 2007) and continuous wavelet transform (Kurnaz and Olmez, 2007) can be used to extract parameters from ultrasound images. Statistical parameters are used for the texture analysis (Haralick et al., 1973). Wavelet is used for the detection of the microcalcification mammograms (Zhang et al., 1998).

Discrete wavelet transform is the model used to explore the frequency component of the images. This method is used in our study to extract the pertinent features.

Corresponding Author: Mohammad Ayache, Department of Biomedical, Faculty of Engineering, Islamic University of Lebanon, BP 30014, Highway Khalde, Lebanon 


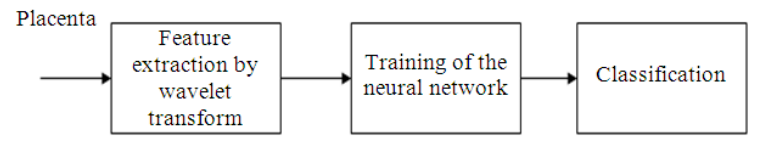

Fig. 1: Classification scheme: Feature extraction by discrete wavelet transform, training of the network and classification of the image

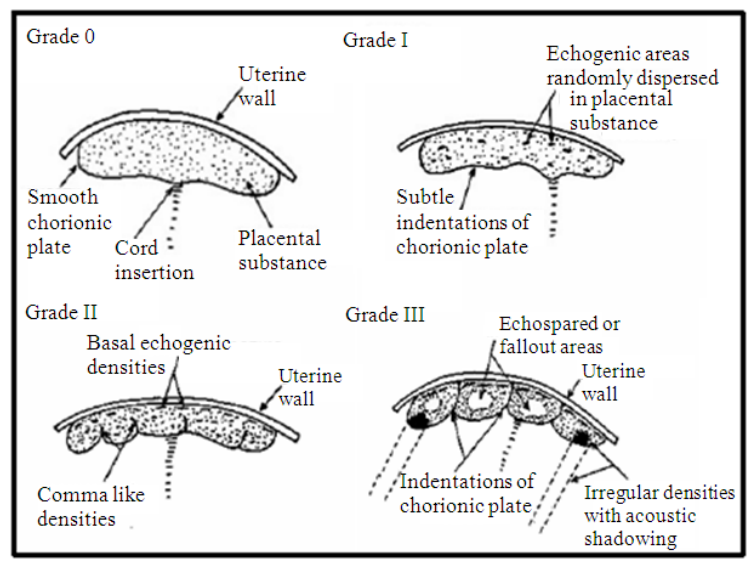

Fig. 2: Diagrammatic version of the grading classifications of the placenta Grades 0-III (Grannum and Hobbins, 1982)

Then the proposed classification approach, using the artificial neural network, needs the extracted features to tell us the grade of placenta. Artificial neural network was used from a long time to characterize and differentiate some signals such as transcranial Doppler signals (Serhatlioglu et al., 2003). The supervised technique, the Multi Layer Perceptron MLP, is used to classify the different tissues in digital mammography (Campos et al., 2007). A complete algorithm of our study is presented in Fig. 1.

Problem statement: Advances in ultrasound technology have allowed a more detailed look at the intrauterine contents and its environment. Until recently, the placenta was evaluated purely to determine its position related to the uterus wall to identify certain premature separation or to subjectively evaluate its maturation. The longitudinal study of placenta has led to the understanding of possible morphologic changes as the placenta matures. From the tenth to the twelfth week of pregnancy the placenta and its margins cannot be clearly delineated. At the end of the first trimester, the chorionic pertinent features from biomedical plate, the core of the placenta and the basal area can be clearly identified. Moreover, the internal structure of it was progressively modified according to the gestational age with changes in the speckle. Finally, this makes that the echostructure was less thin with the detection of large structures with strong ultrasound reflexion changes. On the basis of the ultrasound changes in the placenta through the gestational period, four grades have been previously described (Fig. 2).

Grade 0: The chorionic plate appears as a smooth, straight and well defined unbroken dense line. This can be seen as early as 12 weeks of gestation. The placental structure appears to be homogeneous and devoid of any outstanding echogenic areas. The basal layer also appears homogeneous and of the same texture as the placental substance. This phase is seen in the first and second trimesters.

Grade I: The Grade I placenta manifests the earliest ultrasound changes of placental maturation. The chorionic plate appears as a well-defined unbroken line but presents many subtle undulations. Few scattered echogenic areas appear within the placenta resulting in a loss of homogeneity. There are no echogenicities visible in the basal layer. This grade is usually noted from 30-32 weeks and may persist until term.

Grade II: The maturational changes in the Grade II placental scans involve changes in all three zones. The chorionic plate develops more marked indentations. The placental substance appears to be incompletely divided by the appearance of linear or comma-like echogenic densities that are contiguous to the marked indentations of the chorionic plate. It should be noted that at this phase the linear echogenicities do not reach the basal layer. The echogenic areas within the placenta also appear to be more numerous and slightly larger than those in Grade I. The basal layer becomes punctuated with linear echoes which are arranged with their long axis parallel to the basal layer. These areas are larger and more dense than the echogenic areas, which are randomly dispersed in the placental substance.

Grade III: This late phase represents the mature placenta. The chorionic plate appears interrupted by indentations, which now extend to the basal layer and probably represent the intercotyledonary septa. These are contiguous with linear echogenicities. As a result, the placenta becomes divided into compartments which presumably demarcate the cotyledons. The central portion of these compartments shows echo-spared or fallout areas. In addition, dense, irregularly shaped and echogenic areas appear close to the chorionic plate. The 
echogenic areas from the basal layer become larger, more dense and confluent and in some cases may cause acoustic shadowing.

It should be noted that a given placenta may have simultaneously more than one grade if different sections are examined. In evaluating each scan in this series, the assigned grade corresponded to the most mature portion of the placenta assessed. It is obviously important to visualize as much placental tissue as possible.

In normal pregnancies, it was reported that Grade I appears at 31 weeks of gestation, grade 2 at 36 weeks and Grade III at 38 weeks. But there is no relationship between the gestational age and the grade classification. (Grannum et al., 1979; Grannum, 1983; 1989; Grannum and Hobbins, 1982).

The aim of our studies is to establish an objective placental classification directly from the images. For that, for each image we extract the characteristic parameters and then we apply a classification method to determine the grade. This is a preliminary study to determine automatically the placental grade according to image processing techniques and artificial neural network.

Discrete wavelet transform: The wavelet transform is a very useful tool in the analysis of images. The theory and methods of wavelet analysis are widely presented in books (Chui, 1992; Teolis, 1998). In this study, discrete wavelet analysis is used instead of the continuous wavelet analysis. The discrete wavelet analysis is based on the concept of Multi-Resolution Analysis (MRA) introduced by Mallat (1989). This characterization of the wavelet transform allows the study of an image from the coarse resolution to the fine resolution and the extraction of information in any levels of decomposition.

The (MRA) can be implemented with a two channel filter bank using quadrature mirror filters. The algorithm applies a one- dimensional high and low pass filtering step to both the rows and columns to the input image. Each filtering step is followed by subsampling which results in change in scale.

Transforms in image processing are twodimensional, so we need a few comments on how we implement a separable transform. When a two dimensional transform is separable, we can calculate it by applying the corresponding one dimensional transform to the column first and then to the rows (Fig. 3). At each decomposition level there are four different output images. An approximation of the input image and three detail images. The information contained in the output subbands of the DWT are:
- LL coefficients which correspond to a low pass filter to rows, followed by low pass filter to columns

- HL coefficients which correspond to a low pass filter to rows, followed by high pass filter to columns

- LH coefficients which correspond to a high pass filter to rows, followed by low pass filter to columns

- HH coefficients which correspond to a high pass filter to rows

followed by high pass filter to columns. When a separable transform is applied, only the LL coefficients may need further decomposition. When this decomposition is done at many levels, we get the subband decomposition in Fig. 3.

Artificial neural network: After the calculation of parameters, an artificial neural network is used to classify the images into different grades. A neural network is a general mathematical computing paradigm that models the operations of biological neural systems. The Multi-Layer Perceptron (MLP) is by far the most well known and most popular neural network among all the existing neural network paradigms. This type of neural network is known as supervised network because it requires a desired output in order to be learned (Haykin, 1998; Rumelhart et al., 1986). The goal of this type of network is to create a model that correctly maps the input to the output using historical data so that the model can then be used to produce the output when the desired output is unknown.

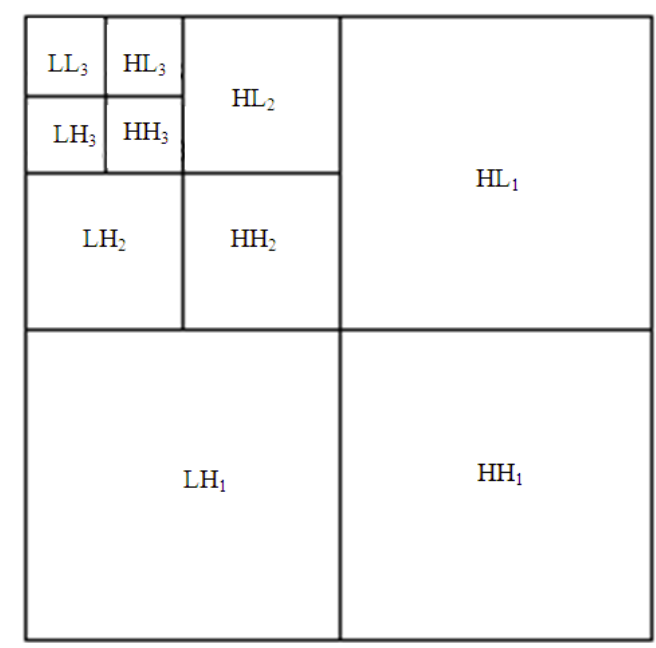

Fig. 3: Passband structure for a two dimensional subband transform with three levels 
This research chooses MLP and its optimization to train and test the data of image registration. The recognition performance of the MLP network will highly depend on the structure of the network and training algorithm. In the current study, back projection algorithm selected to train the network. The number of nodes in input, hidden and output layers will determine the network structure. Furthermore, hidden and output nodes have activation function that will also influence the network performance. The best network structure is normally problem dependent, hence structure analysis has to be carried out to identify the optimum structure. In the current study, the number of input and output nodes were fixed at 10 and 4 respectively, since the images have been divided into 10 subimages each one and the target outputs are 4 grades. Therefore, only the number of hidden nodes and activation functions need to be determined. Mean Squared Error (MSE) will be used to judge the network performance to perform the classification.

For this analysis the images were used to determine the structure of the network. The analysis will be divided into three that are used to determine number of hidden node, type of activation function and sufficient training epoch.

Type of activation functions: Both hidden and output nodes have activation function and the function can be different. There are different functions that are normally used, which are log sigmoid (logsig), tangent sigmoid (tangsig), pure linear (purelin), saturating linear (satlin) and hard limiter (hardlin). For this study, the number of hidden nodes was taken as 5 and the maximum epoch was set to 50. The value of MSE at the final epoch was taken as an indication of the network performance. When the activation functions for the output nodes were selected as log sigmoid and the activations of the hidden nodes were changed, the MSE values as in Table 1 were obtained.

The results in Table 1 suggest that the best hidden node activation function is tansig. The results also revealed that in general sigmoid functions (logsig and tansig) are generally much better than linear functions such as purelin, satlin and hardlin.

Table 1: MSE variation with different activation function of hidden nodes

\begin{tabular}{|c|c|}
\hline Activation function of hidden nodes & MSE \\
\hline Logsig & $2.5854 \times 10^{-11}$ \\
\hline Tansig & $1.9590 \times 10^{-12}$ \\
\hline Purelin & 0.2814 \\
\hline Satlin & 0.0448 \\
\hline Hardlin & 0.1750 \\
\hline
\end{tabular}

A similar study was carried out to determine the most suitable activation function for output nodes. In this case the activation function for hidden nodes was selected as tansig determined by the previous study and the activation function for output nodes were changed. The MSE values were shown in Table 2. The results showed that the most suitable activation function for output nodes was logsig; hence logsig has been selected as the activation function of the output nodes.

Number of hidden nodes: The number of hidden nodes will heavily influence the network performance. Insufficient hidden nodes will cause underfitting where the network cannot recognize the image because there are not enough adjustable parameter to model or to map the input- output relationship. However, excessive hidden nodes will cause overfitting where the network fails to generalize. Due to excessive adjustable parameters the network tends to memorize the inputoutput relation hence the network could map the training data very well but normally fail to generalize. Thus, the network could not map the independent or testing data properly. One way to determine the suitable number of hidden node is by finding the minimum MSE calculated over the testing data set as the number of hidden nodes is varied. Experimentally, the besthidden node activation function is tansig and bestoutput node activation function is logsig.

One way to determine the suitable number of hidden node is by finding the minimum MSE calculated over the testing data set as the number of hidden nodes is varied. The number of hidden nodes was increased from 1-10 and was selected as 5 for the minimum MSE obtained.

Our database is composed of 80 images issued from the 80 studied patients. These images are used to train the neural network system and by taking into account 4 different grades of placenta.

For each image we know the term of pregnancy so the corresponding grade is known. We learn the neural network by choosing the desired output:

- (1000) for class 1

- (0100) for class 2

- $\quad(0010)$ for class 3

- (0001) for class 4

Table 2: MSE variation with different activation function of output nodes

\begin{tabular}{ll}
\hline Activation function of hidden nodes & MSE \\
\hline Logsig & $1.9590 \times 10^{-12}$ \\
Tansig & 0.0385 \\
Purelin & 0.0348 \\
Satlin & $2.5349 \times 10^{-11}$ \\
Hardlin & 0.9000 \\
\hline
\end{tabular}




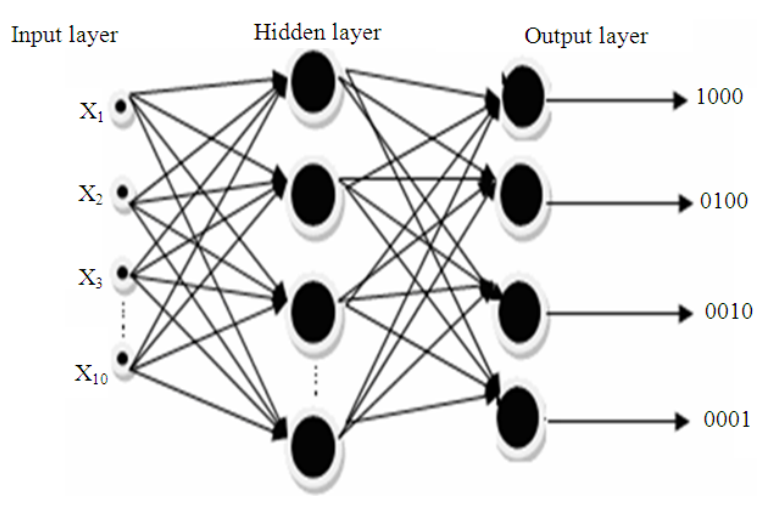

Fig. 4: A neural network (MLP) presented by the input, hidden and output layers

Figure 4 shows a complete schematic for the MLP network used in present study.

\section{MATERIALS AND METHODS}

Eighty normal pregnant volunteers were scanned in Bretonneau Hospital (Tours, France).

The gestational age varied from 21 weeks to 36 weeks. Conventional ultrasound units (Sequoia 512, Siemens; Voluson 530, GE) were used to obtain two dimensional ultrasound images. Only anterior and lateral placentas were considered to avoid any interference with the baby and to allow a more valuable characterization. Data were stored during a sweep of the transducer on the maternal abdomen. The ultrasound images were acquired while applying the probe in an orthogonal orientation to the chorionic plate. All images were acquired in a gray scale mode. Non linear imaging technique was used to acquire the images (Fig. 5).

In this study, discrete wavelet transform at level 3 is applied to the ultrasound placental images to obtain 10 different regions (Fig. 6).

We deduce the parameters which correspond to the variance of each subimage of Fig. 7. These parameters will be different from one grade to another grade of placenta and so they are supposed to be pertinent. The parameters extracted from each region (variance) forms a feature vector for each volunteer. Thus, the supervised training MLP set consists of $\mathrm{N}(\mathrm{N}=60 / 80)$ feature vectors. After the training process, the MLP will classify the ultrasound images into four different grades.

After training and classification, a Principal Component Analysis (PCA) projection is done to visualize the possibility of the separation of all classes. In fact, PCA is used in our case to project the classes from $\mathfrak{R}^{10}$ to $\Re^{2}$.

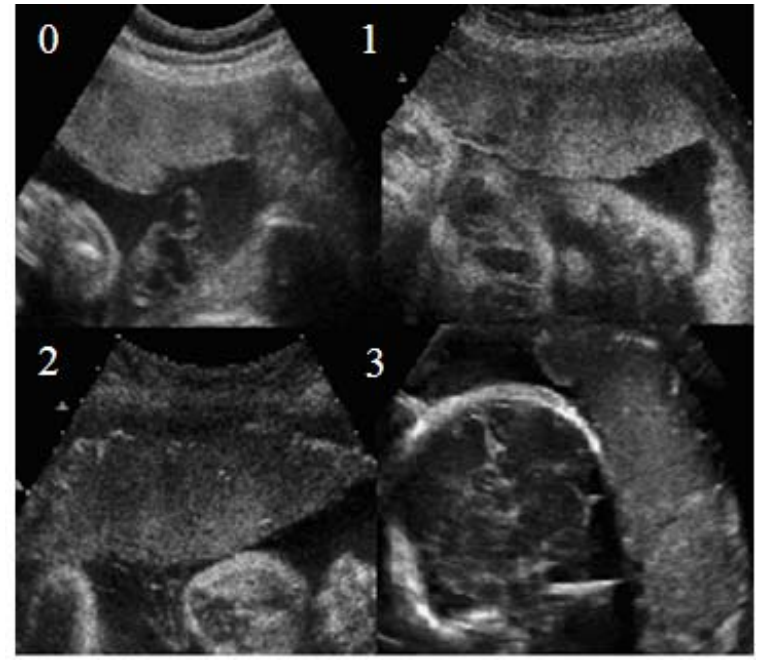

Fig. 5: Different grades of placenta: 0-1-2-3

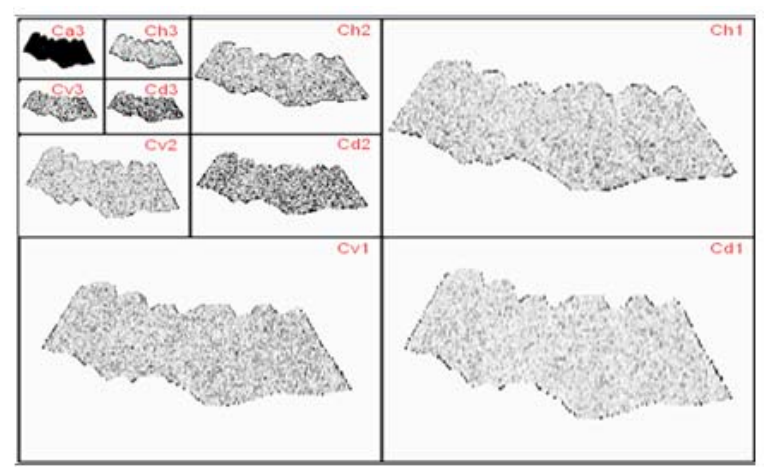

Fig. 6: Wavelet decomposition of placental images at level 3

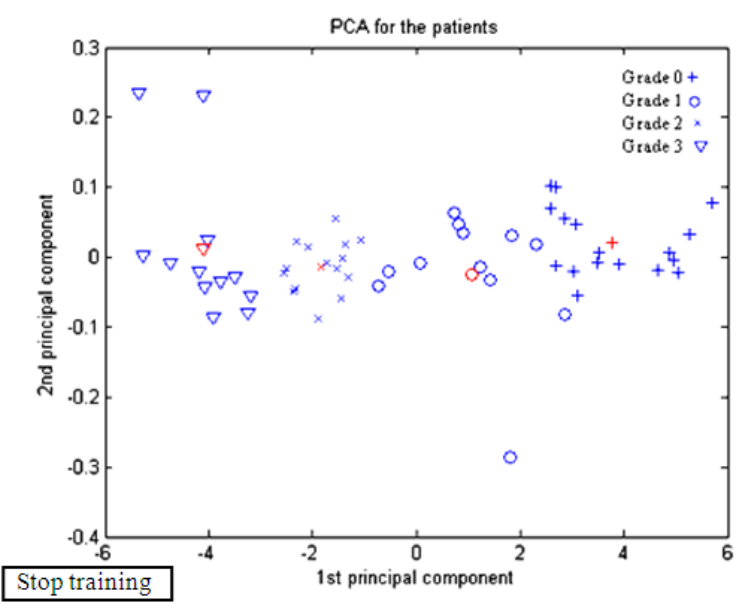

Fig. 7: Principal Component Analysis (PCA) shows the discrimination of the different grades 
Table 3: Confusion matrix shows the misclassified tested images

\begin{tabular}{llllll}
\hline Grade & 0 & I & II & III & Misclassified \\
\hline 0 & 4 & 1 & 0 & 0 & 1 \\
I & 0 & 5 & 0 & 0 & 0 \\
II & 0 & 0 & 5 & 0 & 0 \\
III & 0 & 0 & 0 & 5 & 0 \\
\hline
\end{tabular}

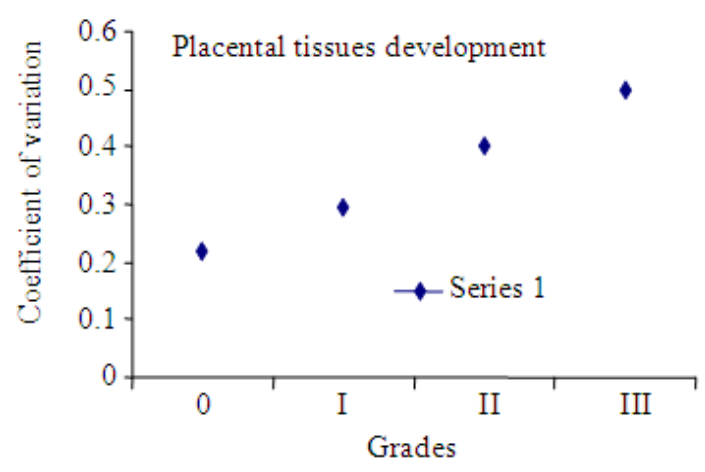

Fig. 8: The evolution of the coefficient of variation of the placenta

Technically speaking, PCA is a linear transformation that transforms the data to a new coordinate system as the greatest variance by any projection of the data comes to lie on the first coordinate (called the first principal component), the second greatest variance on the second coordinate (called the second principal component).

\section{RESULTS}

Figure 7 shows us the results of projection of all classes in $\Re^{2}$. We clearly show the possibility of discrimination between the different classes.

The grade classified in red color corresponds to the center of each grade. 20/80 images, distributed equally over all grades, are used to test the network. The results are presented in Table 3 in a confusion matrix. This matrix shows only one misclassified image in Grade 0 .

We notice that $95 \%$ of images are classified properly. The percentage of error is $5 \%$.

A coefficient of variation shows the longitudinal development of the tissues in terms of the grades (Fig. 8). The coefficient of variation corresponds to the Mean/Standard Deviation (MEAN/STD) (Podobnik et al., 1996).

\section{DISCUSSION}

The study of the placental development and the evolution of the different grades by a method of classification is a very important technique to highlight the longitudinal variation of placental tissues. The goal of this study is to extract different parameters from the different images of placenta then make a more detailed classification for a more accurate diagnosis.

The constitution of the right feature space in image processing applications is highly intuitive. In the literature, there are a variety of feature extraction algorithms applicable to ultrasound images, but a few study have been reported on the placental tissue development. In a previous study (Podobnik et al., 1996), the ultrasonic placental tissue development is characterized by a coefficient of variation according to the week of gestation. No relation exists between the week of gestation and the development of the grade. To study the development of placental tissues according to the different grades, more objective parameters needed to classify the different grades. In this study wavelet transform is used to extract the specific parameters and to classify the different grades of placenta.

\section{CONCLUSION}

The MLP is an effective tool to classify the different grades of placenta. This method is simple and makes an exact classification that helps us to study the transfer function of the placental development and the detection of the anomalies especially those concerning premature birth and intra-uterine growth retardation.

\section{ACKNOWLEDGEMENT}

This project was funded by the research center at the Islamic university of Lebanon and the University of Tours, France.

\section{REFERENCES}

Campos, L.F.A., A.C. Silva and A.K. Barros, 2007. Independent component analysis and neural networks applied for classification of malignant, benign and normal tissue in digital mammography. Methods Inform. Med., 462: 212-215.

Chui, C.K., 1992. An Introduction to Wavelet. Academic Press Professional Inc., San Diego, CA., USA., pp: 266.

Feleppa, E.J., A. Kalisz, J.B.S. Melgar, F.L. Lizzi and T. Liu et al., 1996. Typing of prostate tissue by ultrasonic spectrum analysis. IEEE Trans. Ultrason. Ferroelect. Freq. Control, 43: 609-619.

Grannum, P.A. and J.C. Hobbins, 1982. The placenta. Radiol. Clinics North Am., 20: 353-365.

Grannum, P.A., 1983. Ultrasound examination of the placenta. Clinics Obstetr. Gynecol., 10: 459-473. 
Grannum, P.A., 1989. The placenta. Clinics Diagn. Ultrasound, 25: 203-219.

Grannum, P.A., R.L. Berkowitz and J.C. Hobbins, 1979. The ultrasonic changes in the maturing placenta and their relation to fetal pulmonic maturity. Am. J. Obstet. Gynecol., 133: 915-922.

Haralick, R.M., K. Shanmugan and I. Dinstein, 1973. Texture feature for image classification. IEEE Trans. Syst. Man Cybernet., 3: 610-621.

Haykin, S., 1998. Neural Networks: A Comprehensive Foundation. 2nd Edn., Prentice Hall, ISBN: 0132733501, pp: 842.

Kurnaz, M.N. and T. Olmez, 2007. Tissue segmentation in ultrasound images by using genetic algorithms. Expert Syst. Appli., 34: 2739-2746.

Kurnaz, M.N., Z. Dokur and T. Olmez, 2001. Segmentation of ultrasound images by using an incremental self organized map. Proceedings of the 23rd Annual International Conference of the IEEE Engineering in Medicine and Biology Society, Oct. 25-28, National Technical Information Service, Istanbul, Turkey, pp: 2638-2640.

http://www.dtic.mil/cgi-bin/GetTRDoc?

$\mathrm{AD}=\mathrm{ADA} 412331 \&$ Location $=\mathrm{U} 2 \&$ doc $=$ GetTRDoc.pdf
Kurnaz, M.N., Z.U. Dokur and T. Olmez, 2007. An incremental neural network for tissue segmentation in ultrasound images. Comput. Methods Programs Biomed., 85: 187-195.

Mallat, S., 1989. A theory for multiresolution signal decomposition: The wavelet representation. IEEE Trans. PAMI., 11: 674-693.

Podobnik, M., B. Brayer, S. Ciglar, C. Duin and M. Podgajski, 1996. Ultrasonic fetal and placental tissue characterization and lung maturity. Int. J. Gynecol. Obstetr., 54: 221-229.

Rumelhart, D.E., G.E. Hinton and R.J. Williams, 1986. Learning representations by back-propagating errors. Nature, 323: 533-536.

Serhatlioglu, S., F. Hardalac and I. Guler, 2003. Classification of transcranial doppler signals using artificial neural network. J. Med. Syst., 27: 205-214.

Teolis, A., 1998. Computational Signal Processing with Wavelets. Brikhauser, Boston, ISBN: 10: 0817639098, pp: 352.

Zhang, W., H. Yoshida, R.M. Nishikawa and K. Doi, 1998. Optimally weighted wavelet transform based on supervised training for detection of microcalcifications in digital mammograms. Med. Phys., 25: 949-956. 